\title{
Construction of Vibronic Diabatic Hamiltonian for Excited-State Electron and Energy Transfer Processes
}

\author{
Yu Xie, ${ }^{\dagger}$ Shengshi Jiang, ${ }^{\dagger, \ddagger}$ Jie Zheng, ${ }^{\S}$ and Zhenggang Lan ${ }^{*}, \dagger$ \\ ${ }^{\dagger}$ CAS Key Laboratory of Biobased Materials, Qingdao Institute of Bioenergy and Bioprocess Technology, Chinese Academy of \\ Sciences, Qingdao 266101, China \\ ${ }^{\ddagger}$ University of Chinese Academy of Sciences, Beijing 100049, China \\ ${ }^{\S}$ Industrial Research Institute of Nonwovens \& Technical Textiles, College of Textiles \& Clothing, Qingdao University, Qingdao \\ 266071, China
}

\section{Supporting Information}

ABSTRACT: Photoinduced excited-state electron and energy transfer processes are crucial in biological photoharvesting systems and organic photovoltaic devices. We discuss the construction of a diabatic vibronic Hamiltonian for the proper treatment of these processes involving the projection approach acting on both electronic wave functions and vibrational modes. In the electronic part, the wave function projection approach is used to construct the diabatic Hamiltonian in which both local excited states and charge-transfer states are included on the same footing. For the vibrational degrees of freedom, the vibronic couplings in the diabatic Hamiltonian are obtained in the basis of the pseudonormal modes localized on each monomer site by applying delocalized-to-localized mode projection. This systematic approach allows us to construct the vibronic diabatic Hamiltonian in molecular aggregates.

\section{INTRODUCTION}

Photoinduced excited-state electron and energy transfers (ET and EET) are widely found in biological photoharvesting systems ${ }^{1-4}$ and organic photovoltaic devices. ${ }^{5-8}$ For example, photoinduced charge separation plays an essential role in organic photovoltaic devices. The EET or exciton migration is highly relevant to the high-efficiency solar energy conversion in both photovoltaic materials and biological light-harvesting systems.

Over the last several decades, many theoretical frameworks were developed to describe photoinduced ET and EET processes, including approximate approaches, such as Marcus theory ${ }^{9,10}$ and Förster theory, ${ }^{11}$ and more advanced theoretical descriptions of nonadiabatic dynamics. In these treatments, the use of the diabatic representation is often required, ${ }^{12-14}$ both to provide an intuitive physical picture and to avoid a possible singularity of nonadiabatic couplings in the adiabatic representation. However, most electronic-structure calculations are performed in the adiabatic representation. Thus, it is necessary to seek a suitable approach for the construction of a diabatic model in the basis of ab initio data. The understanding of nonadiabatic dynamics also requires the proper treatment of electron-phonon coupling or vibronic coupling. Thus, the construction of a diabatic Hamiltonian including multiple electronic states and vibronic couplings is rather essential.

In the diabatic representation, the ET/EET processes involve two types of states, i.e., the locally excited (LE) states and the charge-transfer (CT) states. Let us take a dimer system to

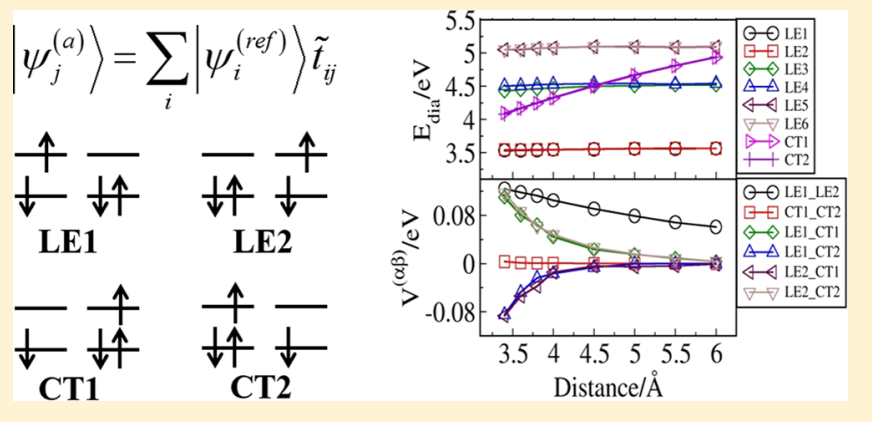

illustrate their definitions. When an electronic transition is localized in one monomer, the resulting electronic state is the LE state. The CT state corresponds to the electronic transition from the occupied orbitals of one monomer to the unoccupied orbitals of the other monomer. In real systems, ET and EET processes may strongly mix with each other. Thus, a proper description of the ET/EET processes requires the construction of the diabatic model Hamiltonian in which LE and CT states are treated on equal footing and vibronic couplings are taken into account.

Generally, there are two categories of methods for performing the diabatization, ${ }^{12}$ i.e., the direct diabatic-state construction ${ }^{4,15-48}$ and the adiabatic-to-diabatic transformation. ${ }^{20,49-105}$ In the first approach, the diabatic states are constructed explicitly before the calculations of the adiabatic electronic wave functions. For example, in the description of excited-state ET and EET processes, one approach is to take individual fragments as building blocks of the whole system. The excitation within an individual fragment is regarded as an LE state. The coupling between two LE states is evaluated using approximate methods. ${ }^{4,15-25}$ However, in this framework, the inclusion of the CT state is quite challenging. ${ }^{24,26}$ It is also possible to "create" the LE or CT states by construction under certain constraints such as electron or density localization.

Received: August 3, 2017

Revised: November 24, 2017

Published: November 27, 2017 
These approaches include the electron-localized broken symmetry wave functions stemming from unrestricted Hartree-Fock (UHF) theory, ${ }^{27-29}$ constrained density functional theory (CDFT), ${ }^{30-35}$ and $\triangle$ SCF-based methods. ${ }^{36-39}$ Recently, the multistate density functional theory (MSDFT) has shown great promise in the construction of diabatic models for large molecular systems and aggregates ${ }^{40-45}$ because this approach constructs the diabatic states directly and allows explicit estimation of interstate couplings.

In the adiabatic-to-diabatic scheme, the wave functions and energies of electronic states are first obtained in the adiabatic representation, and then the diabatic model is constructed via the adiabatic-to-diabatic transformation. In polyatomic systems, the strict adiabatic-to-diabatic transformation can only be defined when all electronic states are included to form the completed Hilbert space. ${ }^{49}$ In practice, it is still possible to define the approximated diabatic states and the relevant adiabatic-to-diabatic transformation within a subspace spanned by the involved electronic states when these states do not strongly couple with other nonrelevant electronic states. ${ }^{49}$ Such diabatization methods are grouped into various classes. ${ }^{12}$ In the simplest system of two equivalent fragments, a two-state model simply shows that the diabatic coupling is the half of the adiabatic energy difference at the minimum adiabatic energy gap. In more complicated situations, the analytical function form of each element in the diabatic Hamiltonian may be assumed first. All parameters in the diabatic model are obtained by fitting of ab initio data ${ }^{12,50,83,84,86-91}$ of the potential energy surface (PES) and other quantities. Although these methods are very practical, analytical expressions of all elements of diabatic Hamiltonian matrix must be assumed. This becomes very difficult in high-dimensional cases. Alternatively, the adiabaticto-diabatic transformation is constructed by the unitary transformation of the electronic basis to eliminate the nonadiabatic couplings. ${ }^{12,49,51-53}$ In addition to a high computational cost, this treatment displays a numerical problem, that is, different integral pathways to the same ending point may result in different adiabatic-to-diabatic transformations. $^{12}$ The third diabatization approach is to construct the wave function itself or the associated physical quantities as smooth functions of nuclear coordinates. ${ }^{12,20,54-80,96-98}$ Several diabatization approaches based on this idea were proposed in the treatment of photoinduced ET or EET in complex systems, which include the generalized Mulliken-Hush (GMH) method $^{65-67}$ and its variants, ${ }^{20,67-72}$ localized diabatization algorithms, ${ }^{73,74}$ and the diabatization method based on maximizing the weight of either the LE or CT configuration. ${ }^{64}$

Although there are many researches on diabatization and various diabatization methods were proposed, we still see that some works focus on this topic in recent years. ${ }^{26,45,80,86-89,92,97,104}$ This implies that diabatization is still very challenging. Recently, Tamura, Burghardt, and coworkers ${ }^{75,76}$ developed a rather practical diabatization method based on the wave function projection approach for timedependent density functional theory (TDDFT). In this approach, ${ }^{75,76}$ the reference wave functions with pure LE and CT characters are defined, and then the projection of adiabatic states onto reference wave functions gives the adiabatic-todiabatic transformation. In fact, more advanced diabatization approaches based on the wave function projection method have been proposed by several groups, ${ }^{54-63,81,82}$ such as the groups of Köppel and Cederbaum, ${ }^{81,82}$ Domcke, ${ }^{54,55}$ Ruedenberg, ${ }^{57,58}$ and Truhlar. ${ }^{59-61}$ These more rigorous approaches involve the diabatization of both molecular orbitals (MOs) and electronic wave functions. Loosely speaking, the method of Tamura, Burghardt, and co-workers ${ }^{75,76}$ may be treated as a simplification of these more rigorous diabatization approaches, and this method is very practical for the exploration of ET/EET processes in large molecular complexes. Here, two closely relevant works that treated photoinduced ET/EET problems should be mentioned. Voityuk ${ }^{80}$ proposed a diabatization scheme based on the transition density in the atom orbital (AO) basis to calculate electronic coupling for photoinduced ET/EET. The quasidiabatic states of reference systems and the adiabatic states of closed stacked systems were all represented by the transition density in the $\mathrm{AO}$ basis. Then the transformation between two sets of transition densities finally defines the adiabatic-to-adiabatic transformation. One tricky point is that the reference states are nonorthogonal, and thus, some additional orthogonalization should be performed in numerical implementations. Köppel and co-workers ${ }^{77-79}$ proposed a method for constructing vibrational diabatic Hamiltonian of homodimers. The delocalized diabatic states of dimer are defined as the symmetric and antisymmetric linear combinations of the localized diabatic states of each monomer. This procedure is very useful in the treatment of homodimers. The extension of this approach to treat many strongly coupled LE and CT states in general stacked systems (with same or different building units) is not fully trivial.

In the construction of a vibronic diabatic Hamiltonian, the other important question is the description of electronphonon coupling or vibronic couplings. For the molecular aggregates, normally the electron-phonon couplings are represented by the spectral density, ${ }^{13}$ which may be extracted from the Fourier transformation of the autocorrelation function reflecting the fluctuations of energies or couplings. ${ }^{106}$ This approach requires long-time molecular dynamic simulations, and great caution must be used in the treatment of detailed balance and other issues. Alternatively, it is also possible to use normal mode coordinates to compute the electron-phonon couplings. $^{25,75,107,108}$

In the current work, we focus on a few of points. (1) Tamura, Burghardt, and co-workers ${ }^{75,76}$ mainly discuss their diabatization method based on TDDFT. We seek a seamless combination of this diabatization approach with additional electronic structure-based methods in which the CIS ${ }^{109}$ (configuration interaction single)-type wave functions can be constructed approximately. For example, we show that the combination of the projection approach and other electronic methods, such as $\operatorname{ADC}(2),{ }^{110,111}$ is straightforward. (2) We wish to carefully examine the numerical performance of the projection-based diabatization method in the treatment of ET and EET problems. Several implementation details are carefully discussed, including the proper selection of the reference wave function and the number of electronic states involved in the diabatization. (3) We discuss a computational protocol based on the vibrational mode projection idea to estimate the vibronic couplings in a diabatic Hamiltonian in the set of pseudonormalmode coordinates localized on each individual building block. For a system with several chromophores, we show that this method not only gives the first-order electron-phonon couplings in the localized pseudonormal-mode coordinates but also provides useful information on the second-order intramode and intermode potential couplings. (4) We pay attention to the dependence of the constructed diabatic model on electronic structure methods. Because we combine the 
projection-based diabatization method with different electronicstructure methods, we can check the dependence of diabatic energies, interstate diabatic couplings, and vibronic couplings on computational methods. Overall, the LE or CT states are localized diabatic electronic states that can be constructed by the projection method in the basis of delocalized adiabatic states. The vibronic coupling is constructed in the localized pseudonormal-mode coordinates. In this way, the vibronic coupling terms of the LE or CT states may only be relevant to the vibrational coordinates of the relevant monomer. This approach shows two advantages. First, after obtaining the diabatic Hamiltonian in a dimer system, it is easy to add more relevant matrix elements to generate the diabatic Hamiltonian of a larger aggregate system. Second, different electronic states may couple to different vibrational modes or "baths" for large systems under this construction. The advantage is that many quantum dissipative dynamical methods can be directly employed to such problems because these theoretical approaches normally require that each state is coupled to its own individual bath. ${ }^{112-114}$

This article is organized as follows: In Section II, theoretical methods are introduced, including the diabatic Hamiltonian, the projection diabatization method, the estimation of vibronic couplings in the basis of localized modes, and electronicstructure calculations. The results and discussion are given in Section III. Our conclusion is presented in the last section.

\section{THEORY}

II.A. Diabatic Hamiltonian. The diabatic electronic Hamiltonian is set up as

$$
V_{e l}^{(d)}=\sum_{\alpha}\left|\psi_{\alpha}\right\rangle V^{(\alpha \alpha)}\left\langle\psi_{\alpha}\left|+\sum_{\alpha \neq \beta}\right| \psi_{\alpha}\right\rangle V^{(\alpha \beta)}\left\langle\psi_{\beta}\right|
$$

where $\left|\Psi_{\alpha}\right\rangle$ and $\left|\Psi_{\beta}\right\rangle$ represent the diabatic states, and LE and CT states are involved. $V^{(\alpha \alpha)}$ denotes the energy of each diabatic state, and $V^{(\alpha \beta)}$ is their interstate coupling.

II.B. Projection Method for Diabatization. We assume that it is possible to find a particular molecular geometry where the adiabatic and diabatic states are identical. At this geometry, adiabatic electronic states should display the pure localized excitonic (LE) or charge-transfer (CT) character. The electronic wave functions at this geometry are defined as the reference states $\left|\Psi_{i}^{(\mathrm{ref})}\right\rangle$.

The adiabatic states $\left|\Psi_{j}^{(a)}\right\rangle$ at the complex's actual geometry can be expanded as the linear combination of diabatic states $\left|\Psi_{j}^{(d)}\right\rangle$, i.e.,

$$
\left|\psi_{j}^{(a)}\right\rangle=\sum_{i}\left|\psi_{i}^{(d)}\right\rangle t_{i j}
$$

where $t_{i j}$ denote the coefficients in the expansion. At the same time, the projection of the adiabatic states onto the reference wave functions gives $\tilde{t}_{i j}$,

$$
\tilde{t}_{i j}=\left\langle\psi_{i}^{(\mathrm{ref})} \mid \psi_{j}^{(a)}\right\rangle
$$

The CI-type wave functions can be expressed in terms of the spin-adapted configuration state functions (CSFs) that are mathematically represented by the linear combination of Slater determinants. The elements of the Slater determinants are molecular orbitals (MOs) that can be further expanded as linear combination of atomic orbitals (AOs). The details of calculations performed to obtain $\tilde{t}_{i j}$ are given in Supporting Information (SI).

In principle, the above equation can be employed for any type of electronic wave function. Similar equations also appear in the more advanced diabatization approaches designed for high-level correlated multireference electronic-structure methods. $^{54-63,81,82}$ In the current work, we only focus on the CIStype excited electronic wave functions due to their low computational cost, even for large systems. The CIS and TDDFT/TDA methods define the CIS-type wave functions directly; ${ }^{109}$ thus, the CI vectors and MO coefficients, as well as the overlap matrix, are directly taken from ab initio calculations to compute the projection according to eq 3 . For TDHF and TDDFT/RPA, we may try to create a pseudowave function from the approximate employment of the Casida's assignment rule. ${ }^{115-120}$ Although such construction is not rigorous, it works in practice because of the small contribution of deexcitation terms. It is also possible to construct the pseudowave function approximately from the $\operatorname{ADC}(2)^{110,111}$ calculation based on its first-order response term.

The above projection results in a nonorthogonal transformation matrix $\tilde{\mathbf{T}}$ with elements $\tilde{t}_{i j}$. The orthogonalization is performed by using ${ }^{59}$

$$
\mathbf{T}=\tilde{\mathbf{T}}\left(\tilde{\mathbf{T}}^{\dagger} \tilde{\mathbf{T}}\right)^{-1 / 2}
$$

where $\mathbf{T}$ is the orthogonal matrix with elements $t_{i j}$. Diabatic states are expanded as

$$
\left|\psi_{j}^{(d)}\right\rangle=\sum_{i}^{N}\left|\psi_{i}^{(a)}\right\rangle t_{j i}^{*}
$$

where $N$ is the number of the states involved. Then, the diabatic Hamiltonian matrix can be easily obtained as

$$
\mathbf{V}_{\mathrm{el}}^{(\mathrm{d})}=\mathbf{T} \mathbf{V}_{\mathrm{el}}^{(\mathrm{a})} \mathbf{T}^{\dagger}
$$

where $\mathbf{V}_{\mathrm{el}}^{(\mathrm{d})}$ is the diabatic electronic Hamiltonian matrix and $\mathbf{V}_{\mathrm{el}}^{(\mathrm{a})}$ is adiabatic electronic Hamiltonian matrix.

The proper preparation of reference states is the first step in the diabatization procedure. In practice, we generate reference states through the electronic-structure calculations of the stacked molecular systems at large intermolecular distances. In principle, in the ideal case, the relevant MO should be completely localized on a single unit. At the same time, the electronic states should display the single LE or CT characteristics; for instance, no mixture between different transition components. Thus, in the ideal case, both MO and electronic states become diabatic. In real situations, such features exist when different molecules are used to build the stacked systems. However, the situation becomes more complicated if two same compounds are used to form the stacked systems. In this case, we found that the relevant MOs become localized, and the "diabatic orbitals" are correctly obtained at the reference geometry in the below systems under study. As a contrast, the localization of the electronic wave functions may not always appear, and the electronic wave functions are still not the "diabatic" ones. Because of the orbital localization, each configuration state function becomes a "diabatic" configuration, and the mixture between different electronic characters is fully dependent on the configurationinteraction (CI) vectors, namely 


$$
\left|\psi_{j}^{(\mathrm{a}, \mathrm{ref})}\right\rangle=\sum_{i}\left|\psi_{i}^{(\mathrm{d}, \mathrm{ref})}\right\rangle c_{i j}
$$

where superscripts (a,ref) and (d,ref) denote adiabatic and diabatic states at reference geometry, respectively. Here, $c_{i j}$ is just the CI vector. Thus, the reversed transformation of the above equations gives the localized diabatic states.

In the following text, the intermolecular distance at the reference configuration is defined as "reference distance" $\left(R_{\text {ref }}\right)$ for simplicity. It is convenient to directly project the adiabatic states at the geometries under study to the reference states at the reference geometry. Previous theoretical works recommended the use of the point-to-point projection procedure. $^{54-62}$ The adiabatic states at geometry $R_{\text {ref }}-\Delta R$ are projected to reference states at $R_{\text {ref, where }} \Delta R$ should be a very small geometry variation toward the target geometry $R$. Then, the adiabatic states at $R_{\text {ref }}-2 \Delta R$ are projected to the diabatic states at $R_{\text {ref }}-\Delta R$. The above steps are repeated until all geometries are covered. We also compare the two procedures in Section III.

II.C. Vibronic Couplings. In this work, we wish to build the pseudonormal modes $\tilde{Q}_{i}$ located at each monomer and then construct the diabatic Hamiltonian in this set of coordinates. For molecular aggregates, we assume that the diabatic state energy $V^{(\alpha \alpha)}$ and the diabatic $V^{(\alpha \beta)}$ coupling are dependent on $\tilde{Q}_{i}$ located at each monomer and the $R$ coordinate representing the distance between the mass centers of the two monomers, i.e.,

$$
\begin{aligned}
& V^{(\alpha \alpha)}(R, \tilde{Q})=V_{0}^{(\alpha \alpha)}(R)+\frac{1}{2} \sum_{i} \tilde{\omega}_{i} \tilde{Q}_{i}^{2}+\sum_{i} \tilde{\kappa}_{i}^{(\alpha)} \tilde{Q}_{i} \\
& V^{(\alpha \beta)}(R, \tilde{Q})=V_{0}^{(\alpha \beta)}(R)+\sum_{i} \lambda_{i}^{(\alpha \beta)} \tilde{Q}_{i}
\end{aligned}
$$

where $\tilde{\omega}_{i}$ and $\tilde{\kappa}_{i}^{(\alpha)}$ are respectively the frequency and the electronic-phonon coupling constant of the corresponding mode, except for intermolecular modes. $V_{0}^{(\alpha \alpha)}$ is zero-order term of diabatic energy. $V_{0}^{(\alpha \beta)}$ and $\lambda_{i}^{(\alpha \beta)}$ are zero and first order terms of the expansion of the diabatic coupling, respectively. In general, electronic couplings $V^{(\alpha \beta)}$ between diabatic states should be strongly dependent on $R$ but depend weakly on $\tilde{Q}_{i}$. Thus, $V_{0}^{(\alpha \beta)}$ should be dominant, while $\lambda_{i}^{(\alpha \beta)}$ in principle should be close to zero.

Several works took the vibronic couplings of the normal modes of the ground-state minimum of each monomer, or its cation/anion, as the modes located at each monomer. ${ }^{25,75,107,108}$ The vibronic couplings are directly computed in the basis of such localized modes, if the diabatic Hamiltonian can be obtained along these modes. For example, Tamura and Burghardt et al. ${ }^{75,76}$ and Köppel et al. ${ }^{77-79}$ used this idea. These above approaches basically assume that the geometries and normal modes remain unchanged from isolated monomers to the building units in the stacked dimer. In reality, some differences may appear, and it should be interesting to check whether such geometry deformation causes the second-order intramode and intermode potential couplings among these localized modes. Of course, these second-order terms may be evaluated by the displacement of different localized normal mode coordinates simultaneously; while the computational cost is large especially for a system with many degrees of freedom. To evaluate this effect, we used a different approach as below.
We took a stacked dimer as a typical example to explain how to define the pseudonormal modes of the dimer system. First, we performed the ground-state optimization of the monomer and computed its normal modes. Then we calculated the ground-state equilibrium geometry of dimer. Next, we overlap the monomer configuration to each building block of the dimer. The proper alignment between them is obtained by the translational and rotational movement of the monomer to minimize the root-mean-square deviation ( $\mathrm{rmsd}$ ) between two coordinate sets. The transformation matrix for molecular rotation is obtained using the Kabsch algorithm. ${ }^{121-123}$ By applying the same rotation on the dimensionless normal coordinates of monomer, we get the pseudonormal modes of the dimer system. Although our approach seems to also use the normal mode of monomer to define the localized modes of the dimer, we also considered the normal modes of the dimer system, which are delocalized modes as well. The transformation between the localized-to-delocalized modes provides us some additional information, see below discussions for details. Mathematically, two sets of normal modes are represented by two high-dimensional vectors; thus, the transformation between them may also be treated as a kind of vector projection. In this sense, the normal-mode projection is performed in the current theoretical treatment. Below we give all details.

We first consider the dimensionless normal mode of the whole dimer system. To construct the dimensionless normal coordinates, the mass-weighted coordinates $\boldsymbol{\xi}$ are introduced as below

$$
\xi=\mathbf{M y}
$$

where $\mathbf{y}$ is the vector of the displacements in Cartesian coordinates and $\mathbf{M}$ is the matrix with elements $M_{i j}=\sqrt{m_{i}} \delta_{i j}$, where $m_{i}$ is the mass of corresponding atom. The transformation between the mass-weighted coordinates $\xi$ and normal coordinates $\mathbf{q}$ are given as below:

$$
\xi=\mathbf{B q}
$$

where the matrix $\mathbf{B}$ is orthogonal and normalized. Now we introduce the dimensionless normal coordinates $\mathbf{Q}$ by

$$
\mathbf{Q}=\mathbf{\Omega q}
$$

with $\Omega_{i j}=\sqrt{\omega_{i}} \delta_{i j}$. We can obtain the relationship between the displacements in Cartesian coordinates and the dimensionless normal coordinates

$$
\mathbf{y}=\mathbf{M}^{-1} \mathbf{B} \boldsymbol{\Omega}^{-1} \mathbf{Q}
$$

The similar transformation can be constructed for the localized pseudonormal modes. Because two sets of modes (pseudonormal modes and dimensionless normal modes of the dimer) are both transferred from the same displacements in Cartesian coordinates; so the delocalized-to-localized mode transformation is as follows:

$$
\tilde{\mathbf{Q}}=\mathbf{P Q}
$$

where $\tilde{\mathbf{Q}}$ and $\mathbf{Q}$ are dimensionless coordinates of localized pseudonormal modes and delocalized modes, respectively. $\mathbf{P}$ is the transformation matrix

$$
\mathbf{P}=\tilde{\mathbf{\Omega}} \tilde{\mathbf{B}}^{-1} \mathbf{M} \mathbf{M}^{-1} \mathbf{B} \boldsymbol{\Omega}^{-1}
$$

Because of the existence of $\tilde{\boldsymbol{\Omega}}$ and $\boldsymbol{\Omega}^{-1}$ in the expression, the transformation matrix $\mathbf{P}$ is not orthogonal. 
Since the pseudonormal modes are constructed from isolated monomer, the number of the delocalized modes is more than that of the localized modes because of six intermolecular vibrational modes. This does not affect the final transformation because the two sets of modes (localized pseudonormal modes and dimensionless normal modes of the dimer) are both transferred from the displacements in Cartesian coordinates.

The electron-phonon couplings in the basis of localized normal coordinates are given by

$$
\tilde{\kappa}_{i}^{(\alpha)}=\sum_{j} \kappa_{j}^{(\alpha)}\left[\mathbf{P}^{-1}\right]_{j i}
$$

where $\tilde{\kappa}_{i}^{(\alpha)}$ and $\kappa_{j}^{(\alpha)}$ are the first-order electron-phonon couplings of the localized pseudonormal modes and the original normal modes of the dimer, respectively. The vibronic coupling of each mode can be characterized by the HuangRhys factors ${ }^{13,14}$

$$
S_{i}^{(\alpha)}=\frac{1}{2}\left(\frac{\kappa_{i}^{(\alpha)}}{\tilde{\omega}_{i}}\right)^{2}
$$

In order to check the dependence of the diabatic electronic couplings $V^{(\alpha \beta)}$ on $\tilde{Q}_{i}$, we define a factor $S_{i}^{(\alpha \beta)}$ similar to the Huang-Rhys factor

$$
S_{i}^{(\alpha \beta)}=\frac{1}{2}\left(\frac{\lambda_{i}^{(\alpha \beta)}}{\tilde{\omega}_{i}}\right)^{2}
$$

The frequencies of localized pseudonormal modes were obtained via

$$
\tilde{\omega}_{i j}^{c}=\sum_{m}\left[\mathbf{P}^{-1}\right]_{m i} \omega_{m}\left[\mathbf{P}^{-1}\right]_{m j}
$$

where $\omega_{m}$ are the frequencies of the dimer. $\tilde{\omega}_{i j}^{c}$ contain the information on the second-order intramode and intermode potential couplings among the pseudonormal modes. In this way, we not only obtained the vibronic (electron-phonon) couplings $\tilde{\kappa}_{i}^{(\alpha)}$ but also examined the possible second-order potential coupling terms.

In the dimer system formed by two equivalent molecules, a simple situation may happen. When the geometry distortion from the isolated monomer to the building unit of the dimer is neglected, all second-order intramode and intermode couplings may vanish completely. Then the symmetric and antisymmetric linear combinations of two equivalent localized modes (with the same frequency) of two monomers define two delocalized modes (also with the same frequency). The frequency remains unchanged in such transformation. This goes back to the simple case discussed by previous works. ${ }^{14,78,79}$

II.D. Computational Details. Three systems were employed to examine the performance of the diabatization scheme, namely, the oligothiophene (quaterthiophene, $\mathrm{OT}_{4}$ ) dimer, the perylene-bisimide (PBI) dimer, and the furan/ dicyanoethylene (DCNE) complex. The ground-state equilibrium geometries were obtained using DFT. TDDFT and spincomponent scaled $\mathrm{ADC}(2)$ [SCS-ADC(2)] methods were used to perform excited-state calculations. The details of the calculations are given in Table S1 in the SI. All DFT and TDDFT calculations were carried out using the Gaussian 09 program. ${ }^{124}$ The TURBOMOLE program ${ }^{125}$ was used to perform the SCS-ADC(2) calculations.

\section{RESULTS AND DISCUSSION}

III.A. Quaterthiophene Dimer. Poly-3-hexylthiophene (P3HT) is widely known for its important role as electron donor in organic photovoltaic devices. ${ }^{126}$ In theoretical studies, the $\mathrm{OT}_{4}$ oligomer is often chosen to understand the photophysical and photochemical properties of $\mathrm{P} \mathrm{HT}^{75,107,127,128}$ The antiparallel $\mathrm{OT}_{4}$ dimer is shown in Figure 1. The frontier MOs are completely delocalized in the

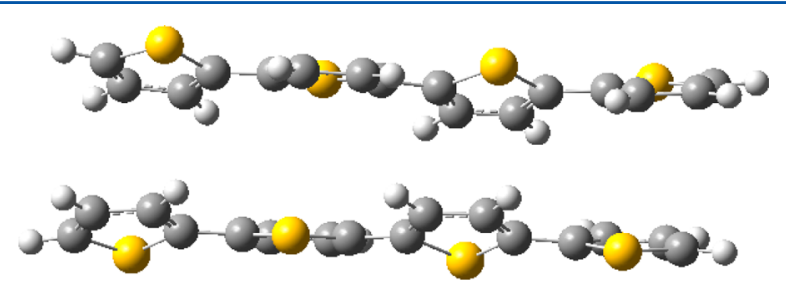

Figure 1. Structure of antiparallel $\mathrm{OT}_{4}$ dimer.

equilibrium configuration (Figure $\mathrm{S} 1$ in the SI). When the distance between two monomers becomes long enough, the relevant molecular orbitals (MO) of the $\mathrm{OT}_{4}$ dimer (Figure S2 in SI) become completely localized in one unit. In the dissociation limit, the two lowest CT states with identical energies are well-defined and do not contain the mixture of different transition components. Therefore, the CT states are diabatic. However, the two lowest adiabatic wave functions are still the mixture of two LE transitions, and this mixture does not vanish, even if we continue to increase the intermolecular distance. In this case, we should use the tricks discussed in Section II.B to generate the diabatic wave functions.

The orientation of $R$ was defined as the distance between two mass centers of two $\mathrm{OT}_{4}$ monomers. As shown in Figures S3 and S4 in the SI, we checked the influence of the $R_{\text {ref }}$ selection and the number of the electronic states included in diabatization. The proper setups give consistent results, except for the minor deviation in the cases with a short $R_{\text {ref }}(8 \AA)$ or a large reference-state number (40 states). To balance the computational accuracy and efficiency, $R_{\text {ref }}=10 \AA$, and 20 states were selected. We also carried out the calculation by using the point-to-point procedure with $\Delta R=0.1 \AA$ and compared the results with those obtained for the direct projection. Because $R$ is a vector, in the calculation we must carefully keep molecule orientation unchanged at different $R$. This approach is necessary because we need to properly calculate overlaps of molecular orbitals of two geometries. As shown in Figure S5 in SI, the results obtained by these two procedures are consistent. For convenience, the direct projection procedure is chosen in the further analysis.

The excitation energies of diabatic and adiabatic states of the $\mathrm{OT}_{4}$ dimer along with the intermolecular distance $R$ are shown in Figure 2. In the diabatic picture, the LE states always appear in a pair (same for the CT states) due to the existence of two equivalent monomers. The excitation energies of the LE states do not depend on $R$, consistent with the intuition that the LE is only relevant to one monomer. The excitation energies of the CT states monotonically increase with $R$ because of the interaction of positive and negative charge densities. The LE1LE2 coupling decreases with $R$, in accord with the $R$ dependence of the adiabatic S1-S2 energy gap. The CT-LE couplings in Figure 2 are comparable and decrease faster than the LE-LE coupling. The CT-CT coupling is nearly zero along with $R$. Overall, the diabatization procedure gives smooth 


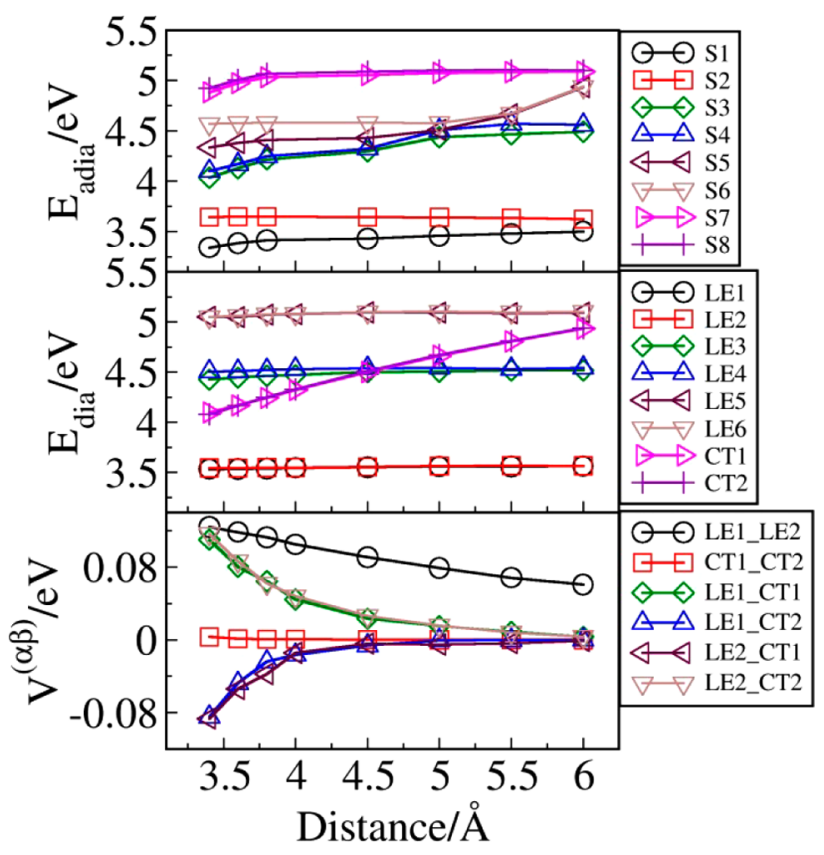

Figure 2. Excitation energies of diabatic states, diabatic couplings, and excitation energies of adiabatic states versus the intermolecular distance of the $\mathrm{OT}_{4}$ dimer. Here, $\tilde{Q}=0$.

diabatic energy curves and works perfectly near $R=4.5-5.5 \AA$, where the CT states cross with the LE states.

Figure 3 gives the Huang-Rhys factor of the diabatic electronic states obtained using the localized normal modes. In all figures, we find that the modes with very low frequencies generally do not give reasonable results, possibly due to anharmonicity. Thus, we do not discuss the contribution of the

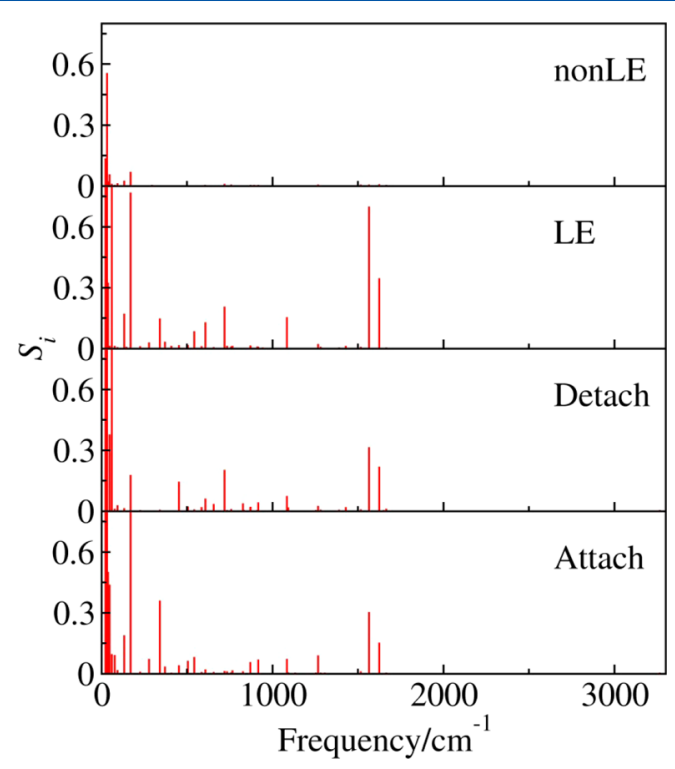

Figure 3. Dependence of Huang-Rhys factor of lowest LE and CT states on the localized pseudonormal modes for the $\mathrm{OT}_{4}$ dimer. $\mathrm{LE}$ denotes that both the LE state and the localized modes are located on the same monomer; nonLE denotes that the LE state and the localized modes are located on different monomers. For the vibronic coupling of the CT state, "Detach" and "Attach" denote the case that the localized modes are relevant to the cationic and anionic part, respectively. low-frequency modes. The localized modes belonging to one monomer give the vibronic couplings only for the LE state localized on the same monomer (Figure 3). It is also clear that the modes with a frequency of approximately $1600 \mathrm{~cm}^{-1}$ give large vibronic couplings. As expected, the localized modes do not result in the vibronic couplings of the LE states relevant to the other monomer. We also observed that the localized normal modes contribute to the vibronic couplings of the CT states because both of the monomers are involved in the formation of the CT state.

In the current system, the molecular aggregates do not cause the dramatic geometry modification from the isolated monomer to the embedded monomer, resulting in the slight frequency difference (relevant to $\tilde{\omega}_{i i}^{c} \tilde{Q}_{i}^{2} / 2$, see Figure 4) for

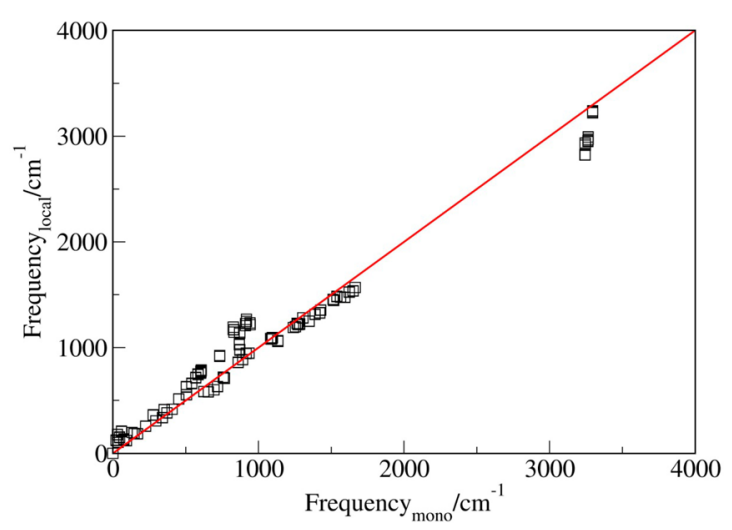

Figure 4. Frequencies (relevant to $\tilde{\omega}_{i i}{ }^{c} \tilde{Q}_{i}{ }^{2} / 2$ ) of localized pseudonormal modes of the $\mathrm{OT}_{4}$ dimer vs. the frequencies of the normal modes of isolated $\mathrm{OT}_{4}$ monomer.

the mode near 900 and $3200 \mathrm{~cm}^{-1}$. The second-order intermode potential couplings are given in Figure 5. They are very small if two modes are localized in different monomers, while such coupling becomes visible if two modes are in the same units. Considering the weak couplings between two monomers, if the LE state of the isolated $\mathrm{OT}_{4}$ monomer is

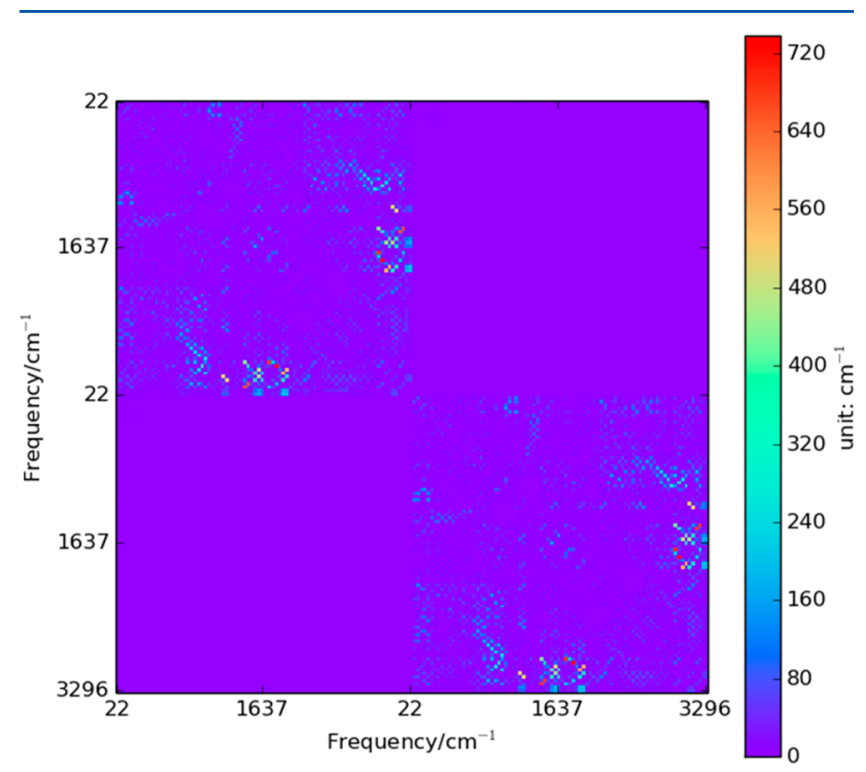

Figure 5. Intermode potential couplings of the localized pseudonormal modes of the $\mathrm{OT}_{4}$ dimer system. 
taken to compute the relevant Huang-Rhys factor, we got the very similar first-order vibronic couplings, and only the very minor differences exist, see Figure 6.

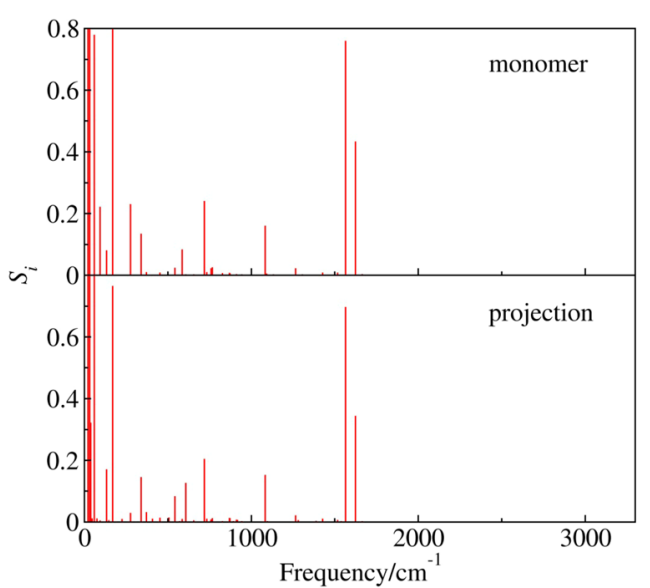

Figure 6. Huang-Rhys factor of LE1 state of the isolated $\mathrm{OT}_{4}$ monomer along the normal modes (upper panel) and the diabatic LE1 state of the $\mathrm{OT}_{4}$ dimer along the localized pseudonormal modes (lower panel).

The dependence of the diabatic couplings $V^{(\alpha \beta)}$ on the localized modes was also checked. As shown in Figure 7, the

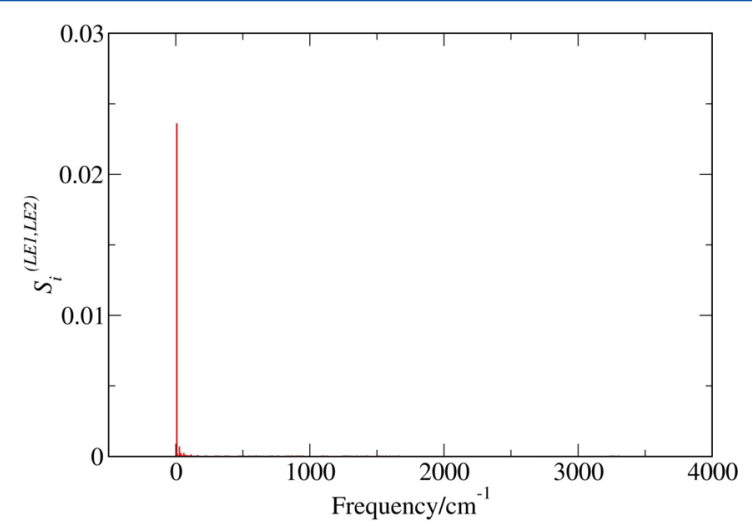

Figure 7. Dependence of the factor $S_{i}^{(\mathrm{LE} 1, \mathrm{LE} 2)}$ characterizing the firstorder vibronic coupling in the diabatic coupling between LE1 and LE2 states on the localized normal modes for the $\mathrm{OT}_{4}$ dimer.

largest value of $S_{i}^{(\mathrm{LE1} 1 \mathrm{LE2})}$ at lowest frequency $7.2 \mathrm{~cm}^{-1}$ is only 0.023 , and the other factors are nearly zero. As expected, the diabatic coupling is dominant by the zero-order term.

III.B. PBI Dimer. The PBI dimer has also received considerable attention in theoretical studies as a representative for various organic semiconductors. ${ }^{25,64,108,129-131}$ At the ground state equilibrium geometry, the PBI dimer displays a $\pi$-stacked face-to-face orientation with a plane-to-plane distance of $3.32 \AA$. Since the monomers are parallel to each other, we define $R$ as the distance between two monomer planes. The dependences of the diabatization on the reference distance and the involved electronic states are given in Figures S6-S9 in the SI. We chose 10 states at $R_{\text {ref }}=8 \AA$ and 14 states at $R_{\text {ref }}=8 \AA$ for $\mathrm{B} 3 \mathrm{LYP}^{132,133}$ and $\omega \mathrm{B} 97 \mathrm{XD}^{134}$ functionals, respectively, in further projection analysis (Figure 8). Similar to the case of $\mathrm{OT}_{4}$, the diabatization obtains good results, and all diabatic energy curves look reasonable. Here, the dependence of the

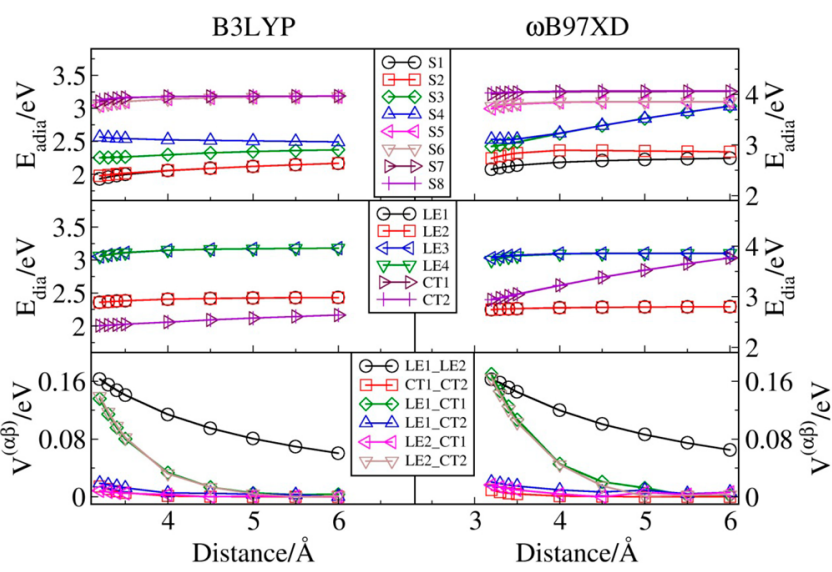

Figure 8. Excitation energies of diabatic states, diabatic couplings, and excitation energies of adiabatic states versus the intermolecular distance of the PBI dimer using B3LYP (left) and $\omega \mathrm{B} 97 \mathrm{XD}$ (right) functionals. Here, $\tilde{Q}=0$.

results on the functional becomes critical. In the diabatic picture, the excitation energies of the CT states calculated at the $\omega \mathrm{B} 97 \mathrm{XD}$ level rise with larger $R$, while those obtained at the B3LYP level remain almost constant due to the failure of TDDFT/B3LYP at long distances. ${ }^{109}$ Although the LE excitation energies are not dependent on $R$ at both levels of theories, the $\omega \mathrm{B} 97 \mathrm{XD}$ level predicts higher excitation energies for the same diabatic LE states than the B3LYP level. However, the diabatic couplings using B3LYP and $\omega$ B97XD display similar curves along intermolecular distances. This seems to indicate that the diabatic coupling is not highly dependent on the computational levels. Ratner and co-workers ${ }^{105}$ found similar phenomenon when they calculated diabatic couplings with GMH theory using CIS, VOA-CIS, and $\omega$ B97X.

As expected, the LE state is only relevant to the modes localized in the same monomer regardless of the functional employed (Figure 9). However, the two functionals gives different vibronic coupling values for the same state. The Huang-Rhys factors at the $\omega \mathrm{B} 97 \mathrm{XD}$ level are larger than those at the B3LYP level because the former functional predicts a larger excited-state gradient than the latter functional (Figure S10 in SI).

It should be noted that Kühn and co-workers ${ }^{25,108}$ have constructed the diabatic Hamiltonian of the PBI dimer by the direct diabatic-state construction approach at the B3LYP/6$311 \mathrm{~g} *$ level. The LE energies were obtained using the electronic structure of the individual monomer, and their couplings were estimated by the Coulomb coupling. Because only the B3LYP functional was used in the monomer calculations, the problem of B3LYP was avoided. They also obtained the vibronic couplings by considering the normal modes of each monomer at the same level of theory. Because the two monomers are not strongly coupled, the frequencies and vibronic couplings in the current work are similar to that of the isolated monomer (Figures S11 and S12 in the SI). Overall, our results for the vibronic couplings of the LE states at the B3LYP level are consistent with their data. ${ }^{25,108}$

III.C. Furan and DCNE. The third model system is a molecular complex that consists of a furan and a DCNE. The molecules are arranged with the $C_{s}$ symmetry in a face-to-face manner.

Beside the direct projection of the adiabatic states at the geometries under study to the reference states at the reference 


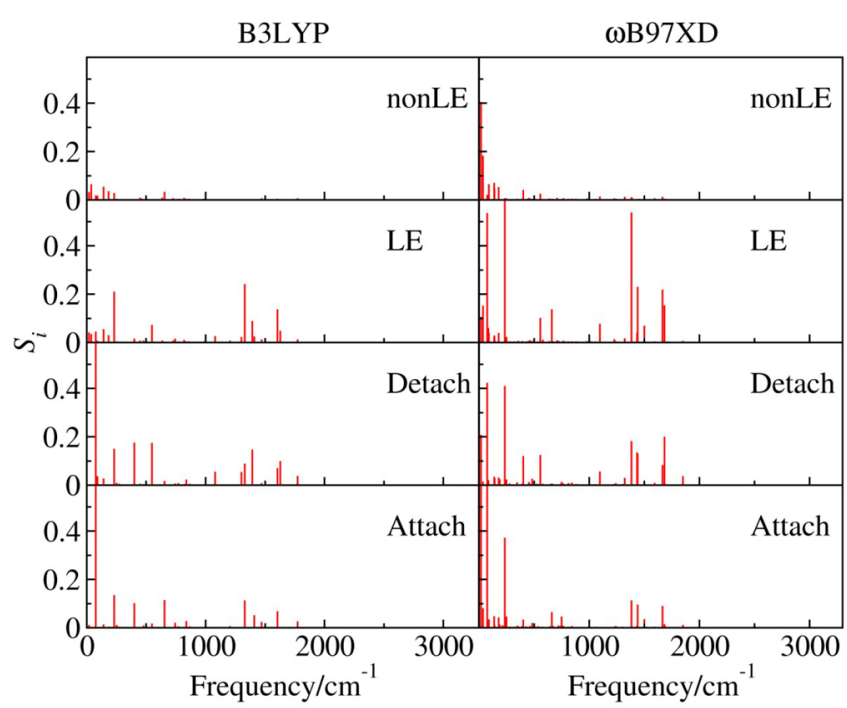

Figure 9. Dependence of Huang-Rhys factors of lowest LE and CT states on the localized pseudonormal modes for the PBI dimer. LE denotes that both the LE state and the localized modes are located on the same monomer; nonLE denotes that the LE state and the localized modes are located on different monomers. For the vibronic coupling of the CT state, "Detach" and "Attach" denote the case that the localized modes are relevant to the cationic and anionic part, respectively.

geometry, we also carried out the calculation by using the point-to-point procedure with $\Delta R=0.1 \AA$. Two different paths were taken in calculations, which always also keeps molecule orientations unchanged. The first path follows the distance of two mass centers of the two molecules, while the second one follows the distance between two molecular planes. At the end, two diabatic Hamiltonians with negligible differences were obtained, see Table S3 in the SI. Thus, the point-to-point projection procedure is stable because we need to follow some particular pathways to perform the diabatization properly.

The excitation energies in the diabatic and adiabatic pictures, as well as the diabatic couplings at the TDDFT ( $\omega$ B97XD) and ADC(2) levels, are shown in Figure 10. In the adiabatic picture, we observe a few potential-energy surface crossings along the intermolecular distance $R$. In the diabatic picture, the state

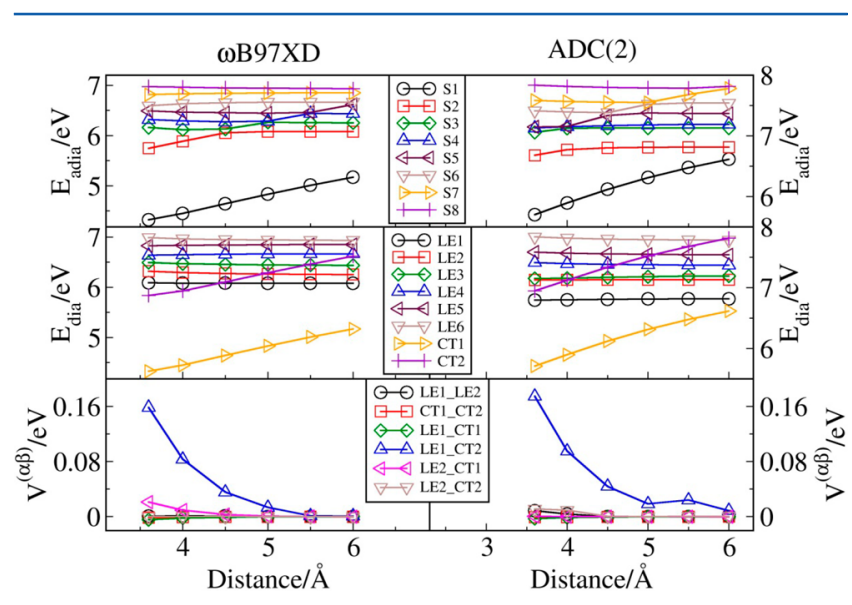

Figure 10. Excitation energies of diabatic states, diabatic couplings, and excitation energies of adiabatic states versus the intermolecular distance of the furan/DCNE complex using $\omega \mathrm{B} 97 \mathrm{XD}$ (left) and $\operatorname{ADC}(2)$ (right) methods. Here, $\tilde{Q}=0$. energy profiles become smooth. With larger $R$, the LE excitation energy curves remain flat, and the CT excitation energy curves rise. Although many features are similar at the TDDFT $(\omega \mathrm{B} 97 \mathrm{XD})$ and $\mathrm{ADC}(2)$ levels, the CT state becomes higher in the $\mathrm{ADC}(2)$ calculations. As a contrast, the TDDFT $(\omega \mathrm{B} 97 \mathrm{XD})$ and $\mathrm{ADC}(2)$ give similar diabatic couplings, similar to the results of the PBI dimer. Furthermore, our diabatization results using $\mathrm{ADC}(2)$ are consistent with the results of previous studies based on other diabatization approaches at the $\operatorname{ADC}(2) /$ aug-cc-pVDZ level. ${ }^{64}$

Many low-lying electronic states only involve the LE transition at the DCNE moiety, and the furan moiety is not relevant at all. Therefore, only the localized modes of the DCNE result in vibronic couplings (Figure 11). However, the CT states are relevant to the transition from furan to DCNE. Thus, the localized modes belonging to both DCNE and furan become relevant to the vibronic couplings.

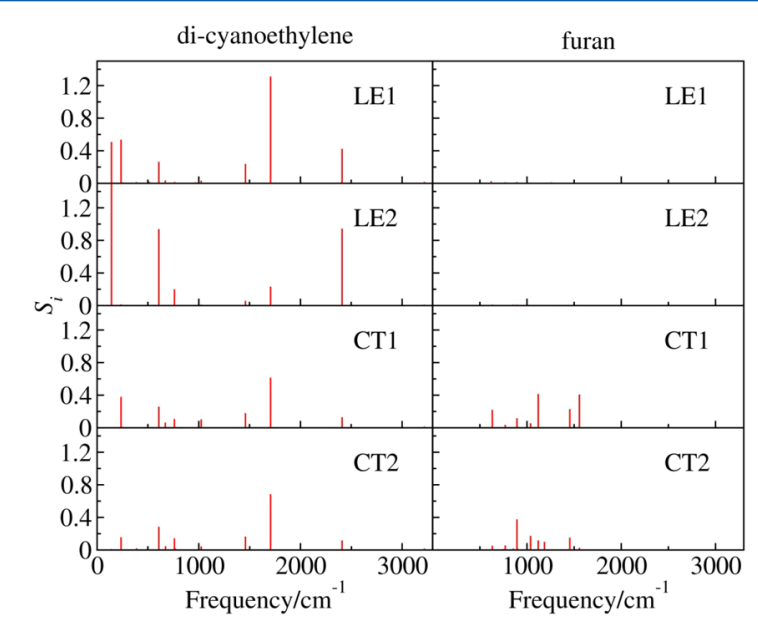

Figure 11. Dependence of Huang-Rhys factors, $S_{i}$, of lowest two LE and $\mathrm{CT}$ states on the localized pseudonormal modes for the furan/ DCNE complex.

\section{CONCLUSION}

In this work, we discussed the construction of the vibronic Hamiltonian in the diabatic representation for the description of the excited-state electron/energy transfer process in stacked dimer systems. This protocol is fully based on the projection methods that not only perform the adiabatic-to-diabatic transformation of electronic states but also provide the delocalization-to-localization transformation of the normal modes and give the vibronic couplings. This provides a vibronic diabatic Hamiltonian on the basis of the pseudonormal modes localized on each monomer.

To validate the scheme, we demonstrate its application to three examples, namely, the $\mathrm{OT}_{4}$ dimer, the $\mathrm{PBI}$ dimer, and the furan/DNCE complex. The performance of the diabatization scheme in these examples confirms that it is appropriate to construct a reasonable diabatic Hamiltonian of stacked molecular systems. We showed that such a diabatization procedure becomes possible when the CIS-type electronic wave function can be constructed, even approximately. We also found that diabatic couplings in all systems under study weakly depend on our chosen methods, although the diabatic energies and intrastate vibronic coupling strengths strongly depended on them. 
Based on the construction of the diabatic model for a dimer system, it is straightforward to extend the dimer model to a large aggregate model. Parameters of various kinds of dimers can be obtained using the same procedure. This provides us a possible approach to the construction of a Hamiltonian including LE and CT states on the same footing for a large aggregate. A similar approach was used within the framework of the renormalized exciton method. ${ }^{135-138}$ We also note that other approaches were proposed for the construction of local vibrational modes. ${ }^{139-141}$ The combination of local-mode construction and diabatization approaches may provide useful and easy-to-use protocols for the construction of the vibronic diabatic Hamiltonian of the large molecular aggregate systems. These efforts will provide a more straightforward approach, even in the black-box manner for the construction of the vibronic diabatic Hamiltonian of complex systems with quantum-chemistry accuracy, which can be further used in the quantum dynamics study of realistic systems in the future.

\section{ASSOCIATED CONTENT}

\section{S Supporting Information}

The Supporting Information is available free of charge on the ACS Publications website at DOI: 10.1021/acs.jpca.7b07737.

Details of diabatization, localized modes, and additional data/figures for the three systems (PDF)

\section{AUTHOR INFORMATION}

\section{Corresponding Author}

*Fax: +86-532-80662778. Tel: +86-532-80662630. E-mail: lanzg@qibebt.ac.cn or zhenggang.lan@gmail.com.

\section{ORCID}

Yu Xie: 0000-0001-8925-6958

\section{Notes}

The authors declare no competing financial interest.

\section{ACKNOWLEDGMENTS}

This work is supported by NSFC projects (Nos. 21503248 and 21673266). This work was also supported by the Natural Science Foundation of Shandong Province for Distinguished Young Scholars (JQ201504). The authors also thank the Supercomputing Center, the Computer Network Information Center, CAS, and the Super Computational Center of CASQIBEBT for providing computational resources.

\section{REFERENCES}

(1) Curutchet, C.; Mennucci, B. Quantum Chemical Studies of Light Harvesting. Chem. Rev. 2017, 117, 294-343.

(2) Blankenship, R. E. Molecular Mechanisms of Photosynthesis; John Wiley \& Sons: 2013.

(3) Mirkovic, T.; Ostroumov, E. E.; Anna, J. M.; van Grondelle, R.; Govindjee; Scholes, G. D. Light Absorption and Energy Transfer in the Antenna Complexes of Photosynthetic Organisms. Chem. Rev. 2017, 117, 249-293.

(4) Cheng, Y. C.; Fleming, G. R. Dynamics of Light Harvesting in Photosynthesis. Annu. Rev. Phys. Chem. 2009, 60, 241-262.

(5) Clarke, T. M.; Durrant, J. R. Charge Photogeneration in Organic Solar Cells. Chem. Rev. 2010, 110, 6736-6767.

(6) Collini, E.; Scholes, G. D. Coherent Intrachain Energy Migration in a Conjugated Polymer at Room Temperature. Science 2009, 323, 369-373.

(7) Song, Y.; Clafton, S. N.; Pensack, R. D.; Kee, T. W.; Scholes, G. D. Vibrational Coherence Probes the Mechanism of Ultrafast Electron Transfer in Polymer-Fullerene Blends. Nat. Commun. 2014, 5, 4933.
(8) Spano, F. C. Excitons in Conjugated Oligomer Aggregates, Films, and Crystals. Annu. Rev. Phys. Chem. 2006, 57, 217-243.

(9) Marcus, R. A. Electron-Transfer Reactions in Chemistry - Theory and Experiment. Rev. Mod. Phys. 1993, 65, 599-610.

(10) Marcus, R. A. On the Theory of Oxidation-Reduction Reactions Involving Electron Transfer.I. J. Chem. Phys. 1956, 24, 966-978.

(11) Förster, T. Zwischenmolekulare Energiewanderung und Fluoreszenz. Ann. Phys. 1948, 437, 55-75.

(12) Domcke, W.; Yarkony, D. R.; Köppel, H. Conical Intersections: Electronic Structure, Dynamics and Spectroscopy; World Scientic: Singapore, 2004.

(13) May, V.; Kühn, O. Charge and Energy Transfer Dynamics in Molecular Systems; Wiley-VCH Verlag GmbH \& Co.: Weinheim, Germany, 2011.

(14) Schröter, M.; Ivanov, S. D.; Schulze, J.; Polyutov, S. P.; Yan, Y.; Pullerits, T.; Kühn, O. Exciton-Vibrational Coupling in the Dynamics and Spectroscopy of Frenkel Excitons in Molecular Aggregates. Phys. Rep. 2014, 567, 1-78.

(15) Alden, R. G.; Johnson, E.; Nagarajan, V.; Parson, W. W.; Law, C. J.; Cogdell, R. G. Calculations of Spectroscopic Properties of the LH2 Bacteriochlorophyll - Protein Antenna Complex from Rhodopseudomonas Acidophila. J. Phys. Chem. B 1997, 101, 4667-4680.

(16) Cory, M. G.; Zerner, M. C.; Hu, X. C.; Schulten, K. Electronic Excitations in Aggregates of Bacteriochlorophylls. J. Phys. Chem. B 1998, 102, 7640-7650.

(17) Krueger, B. P.; Scholes, G. D.; Fleming, G. R. Calculation of Couplings and Energy-Transfer Pathways between the Pigments of LH2 by the ab Initio Transition Density Cube Method. J. Phys. Chem. B 1998, 102, 5378-5386.

(18) Scholes, G. D.; Gould, I. R.; Cogdell, R. J.; Fleming, G. R. Ab Initio Molecular Orbital Calculations of Electronic Couplings in the LH2 Bacterial Light-Harvesting Complex of Rps-Acidophila. J. Phys. Chem. B 1999, 103, 2543-2553.

(19) Jordanides, X. J.; Scholes, G. D.; Shapley, W. A.; Reimers, J. R.; Fleming, G. R. Electronic Couplings and Energy Transfer Dynamics in the Oxidized Primary Electron Donor of The Bacterial Reaction Center. J. Phys. Chem. B 2004, 108, 1753-1765.

(20) Hsu, C. P.; You, Z. Q.; Chen, H. C. H. Characterization of the Short-Range Couplings in Excitation Energy Transfer. J. Phys. Chem. C 2008, 112, 1204-1212.

(21) Madjet, M. E.; Abdurahman, A.; Renger, T. Intermolecular Coulomb Couplings from $\mathrm{Ab}$ Initio Electrostatic Potentials: Application to Optical Transitions of Strongly Coupled Pigments in Photosynthetic Antennae and Reaction Centers. J. Phys. Chem. B 2006, 110, 17268-17281.

(22) Adolphs, J.; Renger, T. How Proteins Trigger Excitation Energy Transfer in the FMO Complex of Green Sulfur Bacteria. Biophys. J. 2006, 91, 2778-2797.

(23) Kawatsu, T.; Coropceanu, V.; Ye, A.; Brédas, J.-L. QuantumChemical Approach to Electronic Coupling: Application to Charge Separation and Charge Recombination Pathways in a Model Molecular Donor-Acceptor System for Organic Solar Cells. J. Phys. Chem. C 2008, 112, 3429-3433.

(24) Yi, Y.; Coropceanu, V.; Brédas, J.-L. Exciton-Dissociation and Charge-Recombination Processes in Pentacene/C-60 Solar Cells: Theoretical Insight into the Impact of Interface Geometry. J. Am. Chem. Soc. 2009, 131, 15777-15783.

(25) Ambrosek, D.; Köhn, A.; Schulze, J.; Kühn, O. Quantum Chemical Parametrization and Spectroscopic Characterization of the Frenkel Exciton Hamiltonian for a J-Aggregate Forming Perylene Bisimide Dye. J. Phys. Chem. A 2012, 116, 11451-11458.

(26) Li, X.; Parrish, R. M.; Liu, F.; Kokkila Schumacher, S. I. L.; Martínez, T. J. An Ab Initio Exciton Model Including Charge-Transfer Excited States. J. Chem. Theory Comput. 2017, 13, 3493-3504.

(27) Newton, M. D. Formalisms for Electron-Exchange Kinetics in Aqueous-Solution and the Role of Abinitio Techniques in Their Implementation. Int. J. Quantum Chem. 1980, 17, 363-391. 
(28) Newton, M. D. Quantum Chemical Probes of Electron-Transfer Kinetics - The Nature of Donor-Acceptor Interactions. Chem. Rev. 1991, 91, 767-792.

(29) Zhang, L. Y.; Friesner, R. A.; Murphy, R. B. Ab Initio Quantum Chemical Calculation of Electron Transfer Matrix Elements for Large Molecules. J. Chem. Phys. 1997, 107, 450-459.

(30) Wu, Q.; Van Voorhis, T. Direct Optimization Method to Study Constrained Systems within Density-Functional Theory. Phys. Rev. A: At., Mol., Opt. Phys. 2005, 72, 024502.

(31) Wu, Q.; Van Voorhis, T. Constrained Density Functional Theory and Its Application in Long-Range Electron Transfer. J. Chem. Theory Comput. 2006, 2, 765-774.

(32) Wu, Q.; Van Voorhis, T. Extracting Electron Transfer Coupling Elements from Constrained Density Functional Theory. J. Chem. Phys. 2006, 125, 164105 .

(33) Wu, Q.; Cheng, C.-L.; Van Voorhis, T. Configuration Interaction Based on Constrained Density Functional Theory: A Multireference Method. J. Chem. Phys. 2007, 127, 164119.

(34) Difley, S.; Van Voorhis, T. Exciton/Charge-Transfer Electronic Couplings in Organic Semiconductors. J. Chem. Theory Comput. 2011, 7, 594-601.

(35) Kaduk, B.; Kowalczyk, T.; Van Voorhis, T. Constrained Density Functional Theory. Chem. Rev. 2012, 112, 321-370.

(36) Gilbert, A. T. B.; Besley, N. A.; Gill, P. M. W. Self-Consistent Field Calculations of Excited States Using the Maximum Overlap Method (MOM). J. Phys. Chem. A 2008, 112, 13164-13171.

(37) Liu, J. Z.; Zhang, Y.; Liu, W. J. Photoexcitation of LightHarvesting C-P-C-60 Triads: A FLMO-TD-DFT Study. J. Chem. Theory Comput. 2014, 10, 2436-2448.

(38) Liu, J. Z.; Zhang, Y.; Bao, P.; Yi, Y. P. Evaluating Electronic Couplings for Excited State Charge Transfer Based on Maximum Occupation Method Delta SCF Quasi-Adiabatic States. J. Chem. Theory Comput. 2017, 13, 843-851.

(39) Kowalczyk, T.; Yost, S. R.; Van Voorhis, T. Assessment of the Delta SCF Density Functional Theory Approach for Electronic Excitations in Organic Dyes. J. Chem. Phys. 2011, 134, 054128.

(40) Cembran, A.; Song, L. C.; Mo, Y. R.; Gao, J. L. Block-Localized Density Functional Theory (BLDFT), Diabatic Coupling, and Their Use in Valence Bond Theory for Representing Reactive Potential Energy Surfaces. J. Chem. Theory Comput. 2009, 5, 2702-2716.

(41) Gao, J. L.; Cembran, A.; Mo, Y. R. Generalized X-Pol Theory and Charge Delocalization States. J. Chem. Theory Comput. 2010, 6, 2402-2410.

(42) Qu, Z. X.; Gao, J. L. Multistate Density Function Theory and the Construction of Diabatic and Adiabatic Potential Energy Surfaces. Chem. J. Chinese U. 2015, 36, 2236-2240.

(43) Gao, J. L.; Grofe, A.; Ren, H. S.; Bao, P. Beyond Kohn Sham Approximation: Hybrid Multistate Wave Function and Density Functional Theory. J. Phys. Chem. Lett. 2016, 7, 5143-5149.

(44) Ren, H. S.; Provorse, M. R.; Bao, P.; Qu, Z. X.; Gao, J. L. Multistate Density Functional Theory for Effective Diabatic Electronic Coupling. J. Phys. Chem. Lett. 2016, 7, 2286-2293.

(45) Grofe, A.; Qu, Z.; Truhlar, D. G.; Li, H.; Gao, J. Diabatic-AtConstruction Method for Diabatic and Adiabatic Ground and Excited States Based on Multistate Density Functional Theory. J. Chem. Theory Comput. 2017, 13, 1176-1187.

(46) Morrison, A. F.; You, Z. Q.; Herbert, J. M. Ab Initio Implementation of the Frenkel-Davydov Exciton Model: A Naturally Parallelizable Approach to Computing Collective Excitations in Crystals and Aggregates. J. Chem. Theory Comput. 2014, 10, 53665376.

(47) Tretiak, S.; Zhang, W. M.; Chernyak, V.; Mukamel, S. Excitonic Couplings and Electronic Coherence in Bridged Naphthalene Dimers. Proc. Natl. Acad. Sci. U. S. A. 1999, 96, 13003-13008.

(48) Morrison, A. F.; Herbert, J. M. Analytic Derivative Couplings and First-Principles Exciton/Phonon Coupling Constants for an $\mathrm{Ab}$ Initio Frenkel-Davydov Exciton Model: Theory, Implementation, and Application to Compute Triplet Exciton Mobility Parameters for Crystalline Tetracene. J. Chem. Phys. 2017, 146, 224110.
(49) Mead, C. A.; Truhlar, D. G. Conditions for the Definition of a Strictly Diabatic Electronic Basis for Molecular-Systems. J. Chem. Phys. 1982, 77, 6090-6098.

(50) Köppel, H.; Domcke, W.; Cederbaum, L. S. Multimode Molecular Dynamics Beyond the Born-Oppenheimer Approximation. Adv. Chem. Phys. 1984, 57, 59-246.

(51) Smith, F. T. Diabatic and Adiabatic Representations for Atomic Collision Problems. Phys. Rev. 1969, 179, 111-123.

(52) Baer, M. Adiabatic and Diabatic Representations for AtomMolecule Collisions - Treatment of Collinear Arrangement. Chem. Phys. Lett. 1975, 35, 112-118.

(53) Baer, M. Adiabatic and Diabatic Representations for AtomDiatom Collisions - Treatment of 3-Dimensional Case. Chem. Phys. 1976, 15, 49-57.

(54) Domcke, W.; Woywod, C. Direct Construction of Diabatic States in the CASSCF Approach - Application to the Conical Intersection of the ${ }^{1} \mathrm{~A}_{2}$ and ${ }^{1} \mathrm{~B}_{1}$ Excited-States of Ozone. Chem. Phys. Lett. 1993, 216, 362-368.

(55) Domcke, W.; Woywod, C.; Stengle, M. Diabatic CASSCF Orbitals and Wave-Functions. Chem. Phys. Lett. 1994, 226, 257-262.

(56) Simah, D.; Hartke, B.; Werner, H. J. Photodissociation Dynamics of $\mathrm{H} 2 \mathrm{~S}$ on New Coupled $\mathrm{Ab}$ Initio Potential Energy Surfaces. J. Chem. Phys. 1999, 111, 4523-4534.

(57) Ruedenberg, K.; Atchity, G. J. A Quantum-Chemical Determination of Diabatic States. J. Chem. Phys. 1993, 99, 3799-3803.

(58) Atchity, G. J.; Ruedenberg, K. Determination of Diabatic States through Enforcement of Configurational Uniformity. Theor. Chem. Acc. 1997, 97, 47-58.

(59) Nakamura, H.; Truhlar, D. G. The Direct Calculation of Diabatic States Based on Configurational Uniformity. J. Chem. Phys. 2001, 115, 10353-10372.

(60) Nakamura, H.; Truhlar, D. G. Direct Diabatization of Electronic States by the Fourfold Way. II. Dynamical Correlation and Rearrangement Processes. J. Chem. Phys. 2002, 117, 5576-5593.

(61) Nakamura, H.; Truhlar, D. G. Extension of the Fourfold Way for Calculation of Global Diabatic Potential Energy Surfaces of Complex, Multiarrangement, Non-Born-Oppenheimer Systems: Application to HNCO(S-0,S-1). J. Chem. Phys. 2003, 118, 6816-6829.

(62) Venghaus, F.; Eisfeld, W. Block-Diagonalization as a Tool for the Robust Diabatization of High-Dimensional Potential Energy Surfaces. J. Chem. Phys. 2016, 144, 114110.

(63) Garcia, V. M.; Reguero, M.; Caballol, R.; Malrieu, J. P. On the Quasidiabatic Character of Average Natural Orbitals. Chem. Phys. Lett. 1997, 281, 161-167.

(64) Liu, W.; Lunkenheimer, B.; Settels, V.; Engels, B.; Fink, R. F.; Köhn, A. A General Ansatz for Constructing Quasi-Diabatic States in Electronically Excited Aggregated Systems. J. Chem. Phys. 2015, 143, 084106.

(65) Cave, R. J.; Newton, M. D. Generalization of the Mulliken-Hush treatment for the calculation of electron transfer matrix elements. Chem. Phys. Lett. 1996, 249, 15-19.

(66) Cave, R. J.; Newton, M. D. Calculation of Electronic Coupling Matrix Elements for Ground and Excited State Electron Transfer Reactions: Comparison of the Generalized Mulliken-Hush and Block Diagonalization Methods. J. Chem. Phys. 1997, 106, 9213-9226.

(67) Yang, C. H.; Hsu, C. P. Multi-State Fragment Charge Difference Approach for Diabatic States in Electron Transfer: Extension and Automation. J. Chem. Phys. 2013, 139, 154104.

(68) Voityuk, A. A.; Rösch, N. Fragment Charge Difference Method for Estimating Donor-Acceptor Electronic Coupling: Application to DNA Pi-Stacks. J. Chem. Phys. 2002, 117, 5607-5616.

(69) Voityuk, A. A. Fragment Transition Density Method to Calculate Electronic Coupling for Excitation Energy Transfer. J. Chem. Phys. 2014, 140, 244117.

(70) Chen, H. C.; You, Z. Q.; Hsu, C. P. The Mediated Excitation Energy Transfer: Effects of Bridge Polarizability. J. Chem. Phys. 2008, $129,084708$.

(71) Hsu, C. P. The Electronic Couplings in Electron Transfer and Excitation Energy Transfer. Acc. Chem. Res. 2009, 42, 509-518. 
(72) You, Z. Q.; Hsu, C. P. Theory and Calculation for the Electronic Coupling in Exciation Energy Transfer. Int. J. Quantum Chem. 2014, 114, 102-115.

(73) Subotnik, J. E.; Yeganeh, S.; Cave, R. J.; Ratner, M. A. Constructing Diabatic States from Adiabatic States: Extending Generalized Mulliken-Hush to Multiple Charge Centers with Boys Localization. J. Chem. Phys. 2008, 129, 244101.

(74) Subotnik, J. E.; Cave, R. J.; Steele, R. P.; Shenvi, N. The Initial and Final States of Electron and Energy Transfer Processes: Diabatization as Motivated by System-Solvent Interactions. J. Chem. Phys. 2009, 130, 234102.

(75) Tamura, H.; Burghardt, I.; Tsukada, M. Exciton Dissociation at Thiophene/Fullerene Interfaces: The Electronic Structures and Quantum Dynamics. J. Phys. Chem. C 2011, 115, 10205-10210.

(76) Tamura, H. Diabatization for Time-Dependent Density Functional Theory: Exciton Transfers and Related Conical Intersections. J. Phys. Chem. A 2016, 120, 9341-9347.

(77) Ottiger, P.; Leutwyler, S.; Köppel, H. S-1/S-2 Excitonic Splittings and Vibronic Coupling in the Excited State of the JetCooled 2-Aminopyridine Dimer. J. Chem. Phys. 2009, 131, 204308.

(78) Ottiger, P.; Leutwyler, S.; Köppel, H. Vibrational Quenching of Excitonic Splittings in H-Bonded Molecular Dimers: The Electronic Davydov Splittings Cannot Match Experiment. J. Chem. Phys. 2012, 136, 174308.

(79) Kopec, S.; Ottiger, P.; Leutwyler, S.; Köppel, H. Vibrational Quenching of Excitonic Splittings in H-bonded Molecular Dimers: Adiabatic Description and Effective Mode Approximation. J. Chem. Phys. 2012, 137, 184312.

(80) Voityuk, A. A. Electronic Couplings for Photoinduced Electron Transfer and Excitation Energy Transfer Computed Using Excited States of Noninteracting Molecules. J. Phys. Chem. A 2017, 121, 54145419.

(81) Pacher, T.; Köppel, H.; Cederbaum, L. S. Quasidiabatic States from Abinitio Calculations by Block Diagonalization of the Electronic Hamiltonian - Use of Frozen Orbitals. J. Chem. Phys. 1991, 95, 66686680.

(82) Pacher, T.; Cederbaum, L. S.; Köppel, H. Approximately Diabatic States from Block Diagonalization of the Electronic Hamiltonian. J. Chem. Phys. 1988, 89, 7367-7381.

(83) Köppel, H.; Gronki, J.; Mahapatra, S. Construction Scheme for Regularized Diabatic States. J. Chem. Phys. 2001, 115, 2377-2388.

(84) Köppel, H.; Schubert, B. The Concept of Regularized Diabatic States for a General Conical Intersection. Mol. Phys. 2006, 104, 10691079.

(85) Pacher, T.; Cederbaum, L. S.; Köppel, H. Adiabatic and Quasidiabatic States in a Gauge Theoretical Framework. Adv. Chem. Phys. 1993, 84, 293-391.

(86) Xie, C.; Malbon, C. L.; Yarkony, D. R.; Guo, H. Dynamic Mapping of Conical Intersection Seams: A General Method for Incorporating the Geometric Phase in Adiabatic Dynamics in Polyatomic Systems. J. Chem. Phys. 2017, 147, 044109.

(87) Zhu, X. L.; Yarkony, D. R. Constructing Diabatic Representations Using Adiabatic and Approximate Diabatic Data - Coping with Diabolical Singularities. J. Chem. Phys. 2016, 144, 044104.

(88) Zhu, X. L.; Malbon, C. L.; Yarkony, D. R. An Improved QuasiDiabatic Representation of the 1, 2, $3{ }^{1}$ A Coupled Adiabatic Potential Energy Surfaces of Phenol in the Full 33 Internal Coordinates. J. Chem. Phys. 2016, 144, 124312.

(89) Zhu, X. L.; Yarkony, D. R. On the Construction of Property Based Diabatizations: Diabolical Singular Points. J. Phys. Chem. A 2015, 119, 12383-12391.

(90) Zhu, X. L.; Yarkony, D. R. Quasi-Diabatic Representations of Adiabatic Potential Energy Surfaces Coupled by Conical Intersections Including Bond Breaking: A More General Construction Procedure and an Analysis of the Diabatic Representation. J. Chem. Phys. 2012, 137, 22 A511.

(91) Yarkony, D. R. On the Adiabatic to Diabatic States Transformation Near Intersections of Conical Intersections. J. Chem. Phys. 2000, 112, 2111-2120.
(92) Hoyer, C. E.; Parker, K.; Gagliardi, L.; Truhlar, D. G. The DQ and DQ Phi Electronic Structure Diabatization Methods: Validation for General Applications. J. Chem. Phys. 2016, 144, 194101.

(93) Yang, K. R.; Xu, X. F.; Truhlar, D. G. Direct Diabatization of Electronic States by the Fourfold-Way: Including Dynamical Correlation by Multi-Configuration Quasidegenerate Perturbation Theory with Complete Active Space Self-Consistent-Field Diabatic Molecular Orbitals. Chem. Phys. Lett. 2013, 573, 84-89.

(94) Hoyer, C. E.; Xu, X.; Ma, D.; Gagliardi, L.; Truhlar, D. G. Diabatization Based on the Dipole and Quadrupole: The DQMethod. J. Chem. Phys. 2014, 141, 114104.

(95) Li, S. H. L.; Truhlar, D. G.; Schmidt, M. W.; Gordon, M. S. Model Space Diabatization for Quantum Photochemistry. J. Chem. Phys. 2015, 142, 064106.

(96) Ichino, T.; Gauss, J.; Stanton, J. F. Quasidiabatic States Described by Coupled-Cluster Theory. J. Chem. Phys. 2009, 130, 174105.

(97) Cave, R. J.; Stanton, J. F. A Simple Quasi-Diabatization Scheme Suitable for Spectroscopic Problems Based on One-Electron Properties of Interacting States. J. Chem. Phys. 2016, 144, 054110.

(98) Cave, R. J.; Stanton, J. F. Block Diagonalization of the Equationof-Motion Coupled Cluster Effective Hamiltonian: Treatment of Diabatic Potential Constants and Triple Excitations. J. Chem. Phys. 2014, 140, 214112.

(99) Paterson, M. J.; Bearpark, M. J.; Robb, M. A.; Blancafort, L.; Worth, G. A. Conical Intersections: A Perspective on the Computation of Spectroscopic Jahn-Teller Parameters and the Degenerate 'Intersection Space'. Phys. Chem. Chem. Phys. 2005, 7, 2100-2115.

(100) Bernardi, F.; Paleolog, S. A. H. D.; Mcdouall, J. J. W.; Robb, M. A. An Effective Hamiltonian-Formalism for the Computation of Diabatic Surfaces. J. Mol. Struct.: THEOCHEM 1986, 31, 23-38.

(101) Bernardi, F.; Robb, M. A. Fragments in Molecules - the Decomposition of Reaction Surfaces into Diabatic Components in the Framework of an Ab-Initio CI Approach. J. Am. Chem. Soc. 1984, 106, 54-58.

(102) Cederbaum, L. S.; Schirmer, J.; Meyer, H. D. Block Diagonalization of Hermitian Matrices. J. Phys. A: Math. Gen. 1989, 22, 2427-2439.

(103) Sirjoosingh, A.; Hammes-Schiffer, S. Diabatization Schemes for Generating Charge-Localized Electron-Proton Vibronic States in Proton-Coupled Electron Transfer Systems. J. Chem. Theory Comput. 2011, 7, 2831-2841.

(104) An, H.; Baeck, K. K. A Practical and Efficient Diabatization That Combines Lorentz and Laplace Functions to Approximate Nonadiabatic Coupling Terms. J. Chem. Phys. 2015, 143, 194102.

(105) Veldkamp, B. S.; Liu, X. L.; Wasielewski, M. R.; Subotnik, J. E.; Ratner, M. A. Molecular Excited States: Accurate Calculation of Relative Energies and Electronic Coupling Between Charge Transfer and Non-Charge Transfer States. J. Phys. Chem. A 2015, 119, 253262.

(106) Valleau, S.; Eisfeld, A.; Aspuru-Guzik, A. On the Alternatives for Bath Correlators and Spectral Densities from Mixed QuantumClassical Simulations. J. Chem. Phys. 2012, 137, 224103.

(107) Huix-Rotllant, M.; Tamura, H.; Burghardt, I. Concurrent Effects of Delocalization and Internal Conversion Tune Charge Separation at Regioregular Polythiophene-Fullerene Heterojunctions. J. Phys. Chem. Lett. 2015, 6, 1702-1708.

(108) Schröter, M.; Kühn, O. Interplay Between Nonadiabatic Dynamics and Frenkel Exciton Transfer in Molecular Aggregates: Formulation and Application to a Perylene Bismide Model. J. Phys. Chem. A 2013, 117, 7580-7588.

(109) Dreuw, A.; Head-Gordon, M. Single-Reference Ab Initio Methods for the Calculation of Excited States of Large Molecules. Chem. Rev. 2005, 105, 4009-4037.

(110) Schirmer, J. Beyond the Random-Phase Approximation - a New Approximation Scheme for the Polarization Propagator. Phys. Rev. A: At., Mol., Opt. Phys. 1982, 26, 2395-2416. 
(111) Trofimov, A. B.; Schirmer, J. An Efficient Polarization Propagator Approach to Valence Electron Excitation Spectra. J. Phys. B: At., Mol. Opt. Phys. 1995, 28, 2299-2324.

(112) Chang, H. T.; Zhang, P. P.; Cheng, Y. C. Criteria for the Accuracy of Small Polaron Quantum Master Equation in Simulating Excitation Energy Transfer Dynamics. J. Chem. Phys. 2013, 139, 224112.

(113) Ai, Q.; Fan, Y. J.; Jin, B. Y.; Cheng, Y. C. An Efficient Quantum Jump Method for Coherent Energy Transfer Dynamics in Photosynthetic Systems under the Influence of Laser Fields. New J. Phys. 2014, 16, 053033 .

(114) Chen, L. P.; Zheng, R. H.; Shi, Q.; Yan, Y. J. Optical Line Shapes of Molecular Aggregates: Hierarchical Equations of Motion Method. J. Chem. Phys. 2009, 131, 094502.

(115) Casida, M. E. Time-Dependent Density Functional Response Theory for Molecules. In Recent Advances in Density Functional Methods; World Scientific: 1995; pp 155-192.

(116) Du, L. K.; Lan, Z. G. An On-the-Fly Surface-Hopping Program JADE for Nonadiabatic Molecular Dynamics of Polyatomic Systems: Implementation and Applications. J. Chem. Theory Comput. 2015, 11, $1360-1374$

(117) Du, L. K.; Lan, Z. G. Correction to "An On-the-Fly SurfaceHopping Program JADE for Nonadiabatic Molecular Dynamics of Polyatomic Systems: Implementation and Applications". J. Chem. Theory Comput. 2015, 11, 4522-4523.

(118) Subotnik, J. E.; Alguire, E. C.; Ou, Q.; Landry, B. R.; Fatehi, S. The Requisite Electronic Structure Theory To Describe Photoexcited Nonadiabatic Dynamics: Nonadiabatic Derivative Couplings and Diabatic Electronic Couplings. Acc. Chem. Res. 2015, 48, 1340-1350.

(119) Barbatti, M.; Pittner, J.; Pederzoli, M.; Werner, U.; Mitrić, R.; Bonačić-Koutecký, V.; Lischka, H. Non-Adiabatic Dynamics of Pyrrole: Dependence of Deactivation Mechanisms on the Excitation Energy. Chem. Phys. 2010, 375, 26-34.

(120) Tapavicza, E.; Tavernelli, I.; Rothlisberger, U. Trajectory Surface Hopping within Linear Response Time-Dependent DensityFunctional Theory. Phys. Rev. Lett. 2007, 98, 023001.

(121) Kabsch, W. A Solution for the Best Rotation to Relate Two Sets of Vectors. Acta Crystallogr., Sect. A: Cryst. Phys., Diffr., Theor. Gen. Crystallogr. 1976, 32, 922-923.

(122) Kabsch, W. A Discussion of the Solution for the Best Rotation to Relate Two Sets of Vectors. Acta Crystallogr., Sect. A: Cryst. Phys., Diffr., Theor. Gen. Crystallogr. 1978, 34, 827-828.

(123) Coutsias, E. A.; Seok, C.; Dill, K. A. Using Quaternions to Calculate RMSD. J. Comput. Chem. 2004, 25, 1849-1857.

(124) Frisch, M. J. T., G, W.; Schlegel, H. B.; Scuseria, G. E.; Robb, M. A.; Cheeseman, J. R.; Scalmani, G.; Barone, V. M. B.; Petersson, G. A.; Nakatsuji, H.; Caricato, M.; Li, X.; Hratchian, H. P.; et al. Gaussian 09; Gaussian, Inc.: Wallingford, CT, 2009.

(125) TURBOMOLE, V6.5, A Development of University of Karlsruhe and Forschungszentrum Karlsruhe GmbH, 1989-2007; TURBOMOLE GmbH, 2007; available from http://www.turbomole. com.

(126) Mehmood, U.; Al-Ahmed, A.; Hussein, I. A. Review on Recent Advances in Polythiophene Based Photovoltaic Devices. Renew. Sust. Energ. Rev. 2016, 57, 550-561.

(127) Spano, F. C.; Silva, C. H- and J-Aggregate Behavior in Polymeric Semiconductors. Annu. Rev. Phys. Chem. 2014, 65, 477500.

(128) Li, H.; Nieman, R.; Aquino, A. J. A.; Lischka, H.; Tretiak, S. Comparison of LC-TDDFT and $\mathrm{ADC}(2)$ Methods in Computations of Bright and Charge Transfer States in Stacked Oligothiophenes. J. Chem. Theory Comput. 2014, 10, 3280-3289.

(129) Schubert, A.; Settels, V.; Liu, W. L.; Würthner, F.; Meier, C.; Fink, R. F.; Schindlbeck, S.; Lochbrunner, S.; Engels, B.; Engel, V. Ultrafast Exciton Self-Trapping upon Geometry Deformation in Perylene-Based Molecular Aggregates. J. Phys. Chem. Lett. 2013, 4, 792-796.
(130) Settels, V.; Liu, W.; Pflaum, J.; Fink, R. F.; Engels, B. Comparison of the Electronic Structure of Different Perylene-Based Dye-Aggregates. J. Comput. Chem. 2012, 33, 1544-1553.

(131) Fink, R. F.; Seibt, J.; Engel, V.; Renz, M.; Kaupp, M.; Lochbrunner, S.; Zhao, H.-M.; Pfister, J.; Würthner, F.; Engels, B. Exciton Trapping in Pi-Conjugated Materials: A Quantum-ChemistryBased Protocol Applied to Perylene Bisimide Dye Aggregates. J. Am. Chem. Soc. 2008, 130, 12858-12859.

(132) Lee, C. T.; Yang, W. T.; Parr, R. G. Development of the ColleSalvetti Correlation-Energy Formula Into a Functional of the ElectronDensity. Phys. Rev. B: Condens. Matter Mater. Phys. 1988, 37, 785-789.

(133) Becke, A. D. Density-Functional Thermochemistry. III. The Role of Exact Exchange. J. Chem. Phys. 1993, 98, 5648-5652.

(134) Chai, J.-D.; Head-Gordon, M. Long-Range Corrected Hybrid Density Functionals with Damped Atom-Atom Dispersion Corrections. Phys. Chem. Chem. Phys. 2008, 10, 6615-6620.

(135) Al Hajj, M.; Malrieu, J. P.; Guihery, N. Renormalized Excitonic Method in Terms of Block Excitations: Application to Spin Lattices. Phys. Rev. B: Condens. Matter Mater. Phys. 2005, 72, 224412.

(136) Zhang, H. J.; Malrieu, J. P.; Ma, H. B.; Ma, J. Implementation of Renormalized Excitonic Method at Ab Initio Level. J. Comput. Chem. 2012, 33, 34-43.

(137) Ma, Y. J.; Liu, Y.; Ma, H. B. A New Fragment-Based Approach for Calculating Electronic Excitation Energies of Large Systems. J. Chem. Phys. 2012, 136, 024113.

(138) Ma, Y. J.; Ma, H. B. Calculating Excited States of Molecular Aggregates by the Renormalized Excitonic Method. J. Phys. Chem. A 2013, 117, 3655-3665.

(139) Xue, R.-J.; Grofe, A.; Yin, H.; Qu, Z.; Gao, J.; Li, H. Perturbation Approach for Computing Infrared Spectra of the Local Mode of Probe Molecules. J. Chem. Theory Comput. 2017, 13, 191201.

(140) Herrmann, C.; Neugebauer, J.; Reiher, M. QM/MM Vibrational Mode Tracking. J. Comput. Chem. 2008, 29, 2460-2470.

(141) Jacob, C. R.; Reiher, M. Localizing Normal Modes in Large Molecules. J. Chem. Phys. 2009, 130, 084106. 